\title{
Musical perception and teaching-learning of French language-culture: Some acoustic and neuroscientific elements for the French music teacher
}

\author{
[Perception musicale et enseignement-apprentissage de la langue-culture \\ française : \\ Quelques éléments acoustiques et neuroscientifiques pour le professeur \\ de musique du français]
}

\author{
Sophie Aubin
}

DOI: 10.18355/XL.2021.14.04.04

\begin{abstract}
The musical nature of the sounds of a modern language emitted in the spoken mode, as well as their rhythmic and melodic combinations, exert a considerable "power" on teaching-learning: they provoke, in the learner, decisive auditory reactions, the variables of which are difficult to control, lead to more or less pleasant sensations, (in) understanding, interpretation, produce meaning. Musical perception is at the heart of successful teaching-learning. A language teacher is first and foremost a language music teacher. A French language-culture teacher is above all and always a French music teacher. Among the multidisciplinary relations of the discipline in which it is located, namely Didactology-didactics of the music of the French language-culture, are acoustics and cognitive neurosciences. Despite the extreme complexity of perceptual and neurological processes, turning to musical acoustics at first and then to musical neurosciences secondly makes it possible to recall and discover essential elements and data likely to be of interest to teaching practices.
\end{abstract}

Key words: musical sounds, language music, musical acoustics, cognitive neuroscience, French language-culture music teacher, musical culture

\section{Résumé}

La nature musicale des sons d'une langue vivante émise sur le mode parlé ainsi que leurs combinaisons rythmiques et mélodiques exercent sur l'enseignementapprentissage un "pouvoir» considérable : elles provoquent, chez l'apprenant, des réactions auditives déterminantes dont les variables sont difficilement contrôlables, entraînent des sensations plus ou moins agréables, une (in)compréhension, une interprétation, produisent du sens. La perception musicale est au cœur du succès de l'enseignement-apprentissage. Un professeur de langue est avant tout et toujours un professeur de musique de langue. Un professeur de langue-culture française est avant tout et toujours un professeur de musique du français. Parmi les relations pluridisciplinaires de la discipline dans laquelle celui-ci se situe, soit la Didactologiedidactique de la musique de la langue-culture française, se trouvent l'acoustique et les neurosciences cognitives. Malgré la complexité extrême des processus perceptifs et neurologiques, se tourner vers l'acoustique musicale dans un premier temps puis les neurosciences musicales dans un second temps permet de rappeler et découvrir des éléments et données essentielles susceptibles d'intéresser les pratiques enseignantes.

Mots-clés : sons musicaux, musique de langue, acoustique musicale, neurosciences cognitives, professeur de musique de langue-culture française, culture musicale

XLinguae, Volume 14 Issue 4, October 2021, ISSN 1337-8384, eISSN 2453-711X 
Le système auditif est plus primal que le système visuel, mais il se structure plus lentement (TROCMÉ-FABRE, 1987, $51)$.

\section{Introduction}

Sans doute n'est-il pas exagéré de considérer, de nos jours, que l'enseignementapprentissage des langues dites " étrangères » en général et du français en particulier, pratiqué en milieux scolaires et universitaires, qu'il s'agisse du français général ou du français sur objectifs spécifiques, est mondialement inscrit certes dans une perspective «actionnelle» mais aussi dans des approches intellectuelles, linguistiques et culturelles, souvent détachées de la sensorialité, de l'affectivité et de la motricité suscitées par les dimensions rythmiques, sonores et musicales de la communication dans la langue cible et dans un environnement donné. Les perceptions visuelles et virtuelles de l'apprenant sont très sollicitées (sauf exercice de compréhension de l'oral exclusivement sonore et auditive), renforcée par l'omniprésence d'écrans collectifs et individuels ${ }^{1}$. Même si les travaux de groupes et en binômes sont très prisés, la motricité des apprenants est le plus souvent toujours réduite à la position assise et attablée, idéale pour l'usage des ordinateurs, tablettes, téléphones et la pratique de l'écrit, le dos tourné à la plupart du groupe-classe. Même si les recherches didactiques contenant des approches artistiques, théâtrales et par conséquent musicales apportent toujours des horizons porteurs (Corral Fullà, Aubin, 2017), la focalisation sur la perception musicale de la langue enseignée est rarement une priorité envisagée dans l'approfondissement et la durée, souvent incompatible avec la précipitation imposée par les délais (nombre d'heures de cours, dates des examens, etc.). Pourtant, il s'agit bien de l'enseignement-apprentissage d'un système musical et instrumental. Le concept de musique de langue pour la légitimation épistémologique de la didactique de la musique du français (DMDF), discipline dont l'appellation a évolué depuis vers la «Didactologie-didactique de la musique de la langue-culture française », a été initialement défini en 1996. Parmi les définitions que nous avons progressivement élaborées, toutes complémentaires, se trouve tout d'abord celle-ci :

«Une musique de langue est un moyen de communication qui consiste dans l'art de combiner des sons, de les organiser dans le temps, de les produire harmonieusement grâce au corps humain, instrument à vent et à percussion et selon des règles spécifiques. » (Aubin, 1996 :72)

Dans cet article, nous verrons certains aspects de la perception musicale des apprenants exposés aux combinaisons sonores du français parlé en tant que langue en cours d'apprentissage, quel que soit leur niveau, en se fondant sur deux questions aussi complexes que fondamentales que nous ne ferons de toute évidence qu'aborder. La première question est à notre sens relativement ancienne : elle consiste à savoir si les sons de langue parlée sont vraiment musicaux. La seconde nous semble plus moderne dans la mesure où, à notre connaissance, du moins en didactique du français, elle n'a pas encore été formulée: sans doute inattendue dans le domaine de la

\footnotetext{
${ }^{1}$ Cette omniprésence varie fortement cependant, si l'on tient compte des multiples écarts et inégalités dus à la fracture numérique.
} 
didactique des langues, elle consiste à savoir si nos apprenants en français ${ }^{2}$ possèdent un " cerveau musicien ». Pour apporter des éléments de réponses à la première, nous rappellerons, dans une perspective à la fois historique et comparative, quelques points de vue acoustiques et phonétiques sur la nature musicale des sons de voix et parole humaines. Pour obtenir un début de réponse à la seconde question, nous nous tournerons vers les neurosciences cognitives spécialisées en musique, à la découverte de ce « cerveau musicien ».

\section{Les sons de langue, produits par la voix humaine sur le mode parlé, sont-ils musicaux ?}

Si l'on se place, comme ce sera le cas tout au long de cet article, dans le domaine de la Didactologie-didactique des musiques de langues-cultures, en codisciplinarité avec la Didactologie-didactique des langues-cultures, l'une des premières démarches est de tenir compte des études comparatives menées en acoustique musicale entre les sons de langue et les sons d'instrument de musique, de manière à mieux connaître la nature de l'objet.

\subsection{Points de vue physiques et objectifs}

Les analyses comparatives entre les sons d'instruments musicaux et des sons vocaux ont leur ancienneté et ne manquent pas de curiosités. Si l'on remonte à la seconde moitié du $\mathrm{XIX}^{\mathrm{e}}$ siècle en acoustique musicale, des techniques de recherches au moyen de résonnateurs et divers appareils mis au point pour analyser les sons musicaux ont été appliquées aux sons de langue, dans le but de déterminer si les sons de la voix humaine étaient musicaux. Des caractéristiques musicales, uniques pour les sons de langues principalement dans le timbre, l'intensité et les harmoniques ont été déduites. Ces extraits de description d'expérience en acoustique musicale que nous citons, menées par le physicien Helmholtz notamment et rapportées par Amédée Guillemin portent sur les sons de langue prononcés :

«Chaque voyelle a (...) un timbre spécial qui résulte de la prédominance d'un son harmonique particulier et de hauteur absolue, de sorte que, sous ce rapport, la voix humaine émet des sons qui se distinguent essentiellement des sons émis par les instruments de musique. Ainsi, la voyelle a a pour son spécifique ou caractéristique le si bémol. Quand nous prononçons le son a, à une hauteur quelconque, c'est le si bémol qui est le son dominant, ou de plus forte résonnance, de la cavité buccale. » (Guillemin,1882 : 238).

D'où le placement de voyelles sur une portée musicale, en fonction de la variation du timbre (Guillemin,1882 : 239) dans leur prononciation. D'où également ce mode « très simple » de vérification du timbre des voyelles, à l'aide d'un diapason :

« Prenez un diapason donnant le si bémol et pendant qu'il vibre, tenez-le en avant de votre bouche; puis prononcez tout bas, sans vous entendre vousmême, les deux voyelles a, o, plusieurs fois et successivement répétées. Vous observerez que le son du diapason est renforcé, toutes les fois que votre bouche fait le mouvement particulier à la voyelle a, tandis qu'il n'est pas modifié par la voyelle o. » (Guillemin, 1882 :239)

${ }^{2}$ Notre démarche est valable pour toute langue et toute musique de langue.

XLinguae, Volume 14 Issue 4, October 2021, ISSN 1337-8384, eISSN 2453-711X 
On peut alors supposer que le recours au diapason, non seulement pour sa musicalité mais pour l'intervention du contrôle auditif du timbre des voyelles aurait eu plus de succès à l'époque chez les enseignants et les apprenants en langues que certains outils de phonétique expérimentale tels que le «palais artificiel » ou le «guide-langue » et autres «jouets scientifiques » qui étaient introduits et conseillés par la phonétique appliquée à l'enseignement des langues à la fin du $\mathrm{XIX}^{\mathrm{e}}$ et au début du $\mathrm{XX}^{\mathrm{e}}(\mathrm{Galazzi}$, 1997 : 175). Le diapason, en tant qu'outil didactico-pédagogique dans le domaine des langues, reste encore inexploré aujourd'hui. Il a toute sa place dans le cadre d'une didactique des musiques de langue, d'une didactique musicale.

Un siècle plus tard, les analyses comparatives et les rapprochements entre les sons d'instrument et les sons de parole, dans le but de dégager la spécificité de chacun, attirent toujours autant l'attention des acousticiens. Leipp (1971: 255 et 282) compare les sonogrammes de sons de divers instruments de musique et de la parole parlée et chuchotée. Le fait d'avoir des appareils de mesure beaucoup plus perfectionnés tend à confirmer la nature musicale des sons de parole, voire même, pourrions-nous affirmer à la relecture de ses propos, à dévoiler une sorte de supériorité acoustique des sons de parole lors de la description des résultats à la découverte de la richesse spectrale obtenue :

«La parole représente des signaux acoustiques très compliqués, qui évoluent rapidement dans le temps. Elle comporte des spectres de raies harmoniques (voyelle) engendrés par les cordes vocales, des explosions plus ou moins nettes, des bruits de souffle à bandes plus ou moins larges, graves ou aiguës. Un mot, une phrase apparaît sur le sonogramme comme une " image » (...). Il s'agit en fait d'une véritable «sténographie » acoustique (...). Ces « signes » apparaissent encore mieux dans la parole chuchotée. » (Leipp, 1989 : 282, cité dans Aubin, $1996: 32$ )

Que ce soit dans les années 70-80 du $\mathrm{XIX}^{\mathrm{e}}$ siècle ou du $\mathrm{XX}^{\mathrm{e}}$, ces acousticiens constatent que l'appareil phonatoire est, du point de vue de son fonctionnement, un véritable instrument à vent, un instrument naturel aux qualités inégalées et inégalables :

«(...) l'organe de la voix chez l'homme, (...) cet instrument de musique naturel, à l'aide duquel nous communiquons nos idées dans les nuances les plus délicates et les plus intimes, instrument si flexible et si complet que les instruments artificiels les plus perfectionnés n'arrivent point à cette diversité de nuances, de timbres, qui permet à la voix humaine d'exprimer les sentiments et les passions les plus variés. » (Guillemin, A. 1882 :259)

« Parmi les instruments à vent, 1 'appareil phonatoire apparaît comme un des plus raffinés, pour peu qu'on sache en "jouer". » (Leipp, 1989 : 285)

La phonétique acoustique, dans les années 1970-1980, abondait dans le même sens avec des analyses comparatives de sons de parole et de sons d'instrument de musique (Landercy, Renard, 1982 : 32). Petar Guberina (Institut de phonétique, Zagreb) avec Paul Rivenc et les équipes du Centre de recherche et d'étude pour la diffusion du français (CREDIF) (Rivenc, 2013) avait déjà mis au point, pour l'enseignementapprentissage des langues, le structuroglobalisme et le verbotonalisme au sein desquels les qualités musicales des sons de langue parlée et la prosodie jouaient et jouent toujours bien évidemment un rôle central et primordial. De leur côté, les 
fondateurs et compositeurs des «musiques nouvelles », dans leur manière de traiter les sons, allaient encore plus loin dans la mesure où ils intégraient naturellement sons et bruits de langue parlée dans leurs compositions musicales (Aubin, 1996 : 54-59). $\mathrm{Au} \mathrm{XXI}{ }^{\mathrm{e}}$ siècle, ces mouvements de rapprochement, distinction, mélanges des sons d'instruments de musique et de parole se sont accentués, rendu possible par les développements informatiques. Ils ont certainement atteint un niveau maximal compte tenu des techniques de traitements professionnels et artistiques de la matière sonore (synthétiseurs vocaux, musicaux, parole artificielle, etc.).

Le professeur de musique de langue-culture française retiendra que les sons et combinaisons sonores du français ont toutes les qualités physiques requises pour entrer dans la catégorie des sons musicaux puisqu'ils possèdent hauteur, timbre, harmoniques, durée, intensité. Pour aller plus loin dans la reconnaissance objective de l'identité musicale des sons de la langue française, nous reproduisons cette conclusion à laquelle nous étions parvenue lors des premières démonstrations de la légitimité épistémologique de la Didactique de la musique du français :

« $\mathrm{Si}$, pour être musical, un son doit être périodique, complexe, pourvu d'un timbre, si les sons apériodiques du français sont des «bruits colorés » et qu'une hauteur peut leur être attribuée au même titre qu'un son de cloche, alors les sons du français (...) sont musicaux. » (Aubin, $1996: 43$ ).

Pour l'enseignant en musique du français, reste à savoir si cette matière sonore diversement produite, reproduite, jouée sera suffisamment attirante voire agréable pour éveiller, chez l'apprenant, dès le début et jusqu'à la fin de son apprentissage, toute l'écoute nécessaire pour développer les mémoires et pratiques musicales requises.

\subsection{Points de vue perceptifs et subjectifs}

Rappelons qu'une description exclusivement physique d'un son ne suffit pas à le définir. La dimension perceptive est tout aussi essentielle. Ce point est d'une importance extrême pour une discipline qui se consacre à l'enseignement d'un système sonore et musicale de communication destiné à être entendu, auditionné, écouté, réécouté, répété, imité, mémorisé, réutilisé, prononcé, articulé, (in)compris, repris un nombre incalculable de fois afin d'aboutir à une bonne maitrise de la communication :

«Stricto sensu, un son est ce que l'on entend quand une onde sonore en propagation dans l'air frappe nos oreilles. Une onde sonore se comporte comme ce que les psychologues appellent un stimulus. Notre réponse au stimulus est le "son" que nous entendons. D'après cette définition, si personne n'écoute (...), il n'y a pas alors de son, mais seulement une onde sonore dans l'air. »(Pierce, $1983: 202$, cité dans Aubin, $1996: 34)$

L'apprenant ${ }^{3}$, lorsqu'il est exposé à la musique d'une langue nouvelle pour lui, reçoit de multiples sensations auditives provoquées par des ondes sonores différentes de celles qui traversent son environnement acoustique habituel. Cette pluie de sons

\footnotetext{
${ }^{3} \mathrm{Il}$ convient de préciser que nous nous référons, dans cet article, à l'apprenant bien entendant, sachant que la prise en compte de la diversité des capacités auditives réelles au sein des groupes d'apprenants relève d'autres recherches qui dépassent le cadre de cet article.
}

XLinguae, Volume 14 Issue 4, October 2021, ISSN 1337-8384, eISSN 2453-711X 
provoque chez lui des réactions, d'une complexité extrême et profonde si l'on a à l'esprit le fait que le nerf auditif est relié au cerveau et à l'ensemble du système nerveux ${ }^{4}$. Sauf élève qui extérioriserait et arriverait à exprimer toutes ses réactions, celles-ci sont bien difficiles à décrypter par l'enseignant. Dès lors, il est possible d'affirmer que la sensibilité auditive individuelle de l'apprenant, que l'expérience soit vécue de manière positive ou négative, est généralement ignorée ou banalisée.

L'apprenant passe par conséquent forcément par des étapes psychophysiologiques troublées, riches en perceptions auditives. Si, en outre, il a été forgé, depuis les débuts de sa scolarité, par des approches exclusivement linguistiques, peu artistiques et non musicales des langues qu'il a déjà étudiées, il est loin d'être prédisposé à la perception de la musicalité de la matière « français » qu'il a choisie ou qu'il doit obligatoirement apprendre. La langue française, nouvelle langue à ses yeux, ses oreilles, son appareil audio-phonatoire, son corps, son esprit se présente pourtant comme un système musical de communication. Chaque phrase travaillée doit bien avoir une musique et un phrasé capables de produire un sens qui sera perçu, interprété. Il est probable que le soin particulier apporté à la musique de chaque phrase par l'enseignant permet de se rapprocher de cette sensibilité auditive de l'apprenant, particulièrement sollicitée et activée.

Aux habitudes éducatives de chaque acteur de l'enseignement-apprentissage, à cette sensibilité auditive, vient s'ajouter une autre variable difficile à contrôler : les goûts musicaux pour les langues que nous entendons.

En effet, l'ensemble résultant de combinaisons de rythmes, mélodies, sons d'une langue parlée, produit avec plus ou moins de justesse par une voie humaine, est soumis à un processus de perception-réception auditive commun à tout genre musical, toute réalisation dans une musique de langue reconnue ou inconnue par le récepteurauditeur. La sensation peut alors être plus ou moins agréable, voire plus ou moins supportable selon de multiples facteurs personnels, culturels, situationnels, mémoriels, etc. Même si la langue française par exemple est généralement considérée comme une langue "musicale », agréable à entendre, la réalité perceptive est extrêmement contrastée. L'enseignant doit conjuguer avec un spectre de goûts musicaux qui peut aller de la détestation inavouée ou inavouable (la matière serait alors imposée par l'institution scolaire ou répondre à des besoins professionnels ou présente dans l'emploi du temps pour tout autre mesure prise dans le cadre familial ou d'une politique linguistique) à la véritable passion.

Nous avons émis l'hypothèse d'une diminution de l'impact de cette variable par une exposition auditive naturelle, dès la plus petite enfance, aux genres de musique de langue en provenance d'horizons culturels les plus variés, non seulement pour la préparation à l'apprentissage de langues mais aussi, voire surtout, pour la familiarisation précoce avec les cultures du monde, la musique d'une langue étant indissociable de sa culture (Aubin, 2008). Cela nous ramène aux systèmes éducatifs et habitudes prises dans les institutions scolaires, le plus souvent peu orientées vers une formation musicale intégrale et intégrée, à tel point que l'établissement du profil musical d'un apprenant en français serait aussi insolite que laborieux.

\footnotetext{
${ }^{4}$ Suite au brusque développement des cours à distance dû à la COVID-19, il serait d'ailleurs intéressant d'effectuer des études comparatives sur cette sensorialité auditive en présentiel et en distanciel dans les cours de langues.
} 
Toutefois, il est certain que de nos jours, le professeur de musique de langue-culture française possède assez d'éléments pour fonder son action didactico-pédagogique sur le fait que les sons de langue et leurs combinaisons, produits par la voix humaine sur le mode parlé, sont musicaux.

\section{L'apprenant en langue différente a-t-il un « cerveau musicien »?}

$\mathrm{Si}$, aux $\mathrm{XIX}^{\mathrm{e}}$ et $\mathrm{XX}^{\mathrm{e}}$ siècles, la didactique des langues a tissé avec la phonétique et l'acoustique des communications interdisciplinaires précieuses, pour ne citer que ces domaines, il semble bien qu'entre les $\mathrm{XX}^{\mathrm{e}}$ et $\mathrm{XXI}^{\mathrm{e}}$ siècle, ses relations avec les neurosciences représentent un enjeu comparable. Nous remarquons que certains chercheurs en neurosciences cognitives sont, de nos jours, tout aussi attirés par les analyses comparatives entre les sons de langue et les sons d'instruments musicaux que les chercheurs en acoustique musicale et en phonétique des siècles derniers que nous venons de citer. Ces perspectives interdisciplinaires intéressent vivement la didactologie-didactique des langues-culture et, qui plus est, la didactologie-didactique des musiques de langue.

\subsection{Petar Guberina (2013-2005) et les neurosciences cognitives actuelles}

En 2018, Jacques Cortès, linguiste et didacticien en langue-culture française, a réalisé une étude approfondie, dont on ne peut que recommander la lecture, des apports des neurosciences cognitives à la Didactologie-didactique de la langue-culture française. Après examen de nombreux travaux qui ont jalonné le $\mathrm{XX}^{\mathrm{e}}$ siècle et de travaux plus récents et très actuels, l'auteur constate que la contribution de certains chercheurs à cet édifice a été paradoxalement oubliés (Ch. Bally, P. Guberina, H. Van Lier) et que la Didactologie-didactique de la langue-culture française ne possède, en matière de prise en compte des neurosciences, aucun véritable «retard» du point de vue scientifique ; elle a même une longueur d'avance considérable, dans la mesure où, dès les années 50-70, les thèses et travaux de Petar Guberina ont été scientifiquement intégrés à la méthodologie de la langue française et de bien d'autres langues européennes (voir Supra 1.1.) donnant des résultats excellents, notamment dans la qualité de l'acquisition des systèmes rythmique, mélodique et phonique du français :

« Guberina considérait que le fonctionnement structural de la langue était un fait d'ordre biologique au départ en soulignant fortement que « c'est parce que le cerveau fonctionne de façon structurale que la langue présente une organisation structurale. Jean-Pierre Changeux et Stanislas Dehaene ${ }^{5}$ nous disent, sinon la même chose, du moins pourraient sûrement considérer cette idée de Guberina comme très proche de la leur. Vraie ou non prouvée, cette affirmation fut d'évidence prémonitoire (...). » (Cortès, $2018: 58$ )

Cette adéquation entre le verbotonalisme et les neurosciences cognitives du $\mathrm{XXI}^{\mathrm{e}}$ siècle est par conséquent hautement pertinente pour l'enseignant en musique de langue : en suivant la méthode verbo-tonale au moins dans ses grandes lignes dont la priorité aux éléments prosodiques, la prise en compte de la phonétique combinatoire et de la prononciation nuancée, l'importance du temps et de la patience nécessaire pour obtenir des résultats durables, etc. (Renard, 1979, Billières, 2021, Zarekar,

\footnotetext{
${ }^{5}$ CHANGEUX, J-P. (1983). L'homme neuronal. Paris : Fayard, Le temps des sciences. DEHAENE, S. (Dir.) (2011). Apprendre à lire. Des sciences cognitives à la salle de classe. Paris : O. Jacob.
}

XLinguae, Volume 14 Issue 4, October 2021, ISSN 1337-8384, eISSN 2453-711X 
Rahmatian, R. 2017), l'enseignant en musique du français a l'assurance d'aller dans le bon sens du fonctionnement cérébral et d'une bonne éducation et rééducation de la perception auditive des sons de la langue apprise. En agissant prioritairement sur l'éducation de l'oreille, le verbo-tonalisme contribue à former le «cerveau musicien » des apprenants. Cela étant acquis, il est important de savoir si les neurosciences cognitives actuelles du domaine spécifiquement musical peuvent nous apporter des éléments pertinents pour notre didactique spécifique.

\subsection{Cerveau musicien et cerveau linguistique}

Nous empruntons l'expression «cerveau musicien» à l'ouvrage consacré aux recherches en neurosciences développées dans le domaine musical, intitulé «Le cerveau musicien. Neuropsychologie et psychologie cognitive de la perception musicale » (Lechevalier, Platel, Eustache, 2010). Selon Hervé Platel (2010 : 291), un «cerveau musicien », s'il existe, est «à l'image d'un cerveau spécialisé dans le traitement du langage, avec des régions corticales spécialisées dans la perception et la mémorisation de la musique ». Ce qui permet à un enseignant en musique de langue de réaliser d'emblée à quel point le cerveau de chaque apprenant débutant en musique de la langue française possède déjà, en tant que spécialiste en traitement du langage humain, un grand réservoir de régions corticales spécialisées dans la perception et la mémorisation musicales, qu'il s'agit de solliciter autrement, afin qu'il puisse percevoir et mémoriser une nouvelle musique de langue et combien il serait regrettable de considérer cet apprenant dépourvu de «cerveau musicien » ou de terrain propice à son développement.

De façon générale, les enseignants et apprenants en langue s'adressent consciemment quasi exclusivement à un «cerveau linguistique», souvent imaginé de façon compartimenté, localisé dans une zone intellectuelle supérieure. Or, si les sons de langue sont musicaux et que l'ensemble du système linguistique d'une langue repose sur son propre système musical, on se situe dans ce cas en permanence dans une sorte de « dialogue de sourds perceptif » entre un enseignant et ses apprenants, dialogue qui entretient, de plus, la «surdité » naturelle de l'apprenant aux éléments musicaux (rythmes, timbres, intonations) qui n'existent pas de la même manière dans la ou les langue(s) déjà maîtrisée(s). En revanche, les enseignants et apprenants en musique de langue ont une probabilité plus grande de s'adresser au "cerveau musicien » en priorité, sans délaisser pour autant le « cerveau linguistique » et de se situer dans cette plasticité qui correspond au fonctionnement neurocognitif. En effet, des études récentes nous apprennent ou confirment, grâce aux techniques de neuro-imagerie par exemple, qu'il faut définitivement « sortir d'une vision localisationniste du fonctionnement cérébral », le plus important étant la capacité de «connectivité » et la plasticité du cerveau (Platel, 2017 : 147), offrant la possibilité d'avoir une influence sur le développement neuronal du cerveau, ce qui va dans le sens de la (re)construction structuro-globale de la perception auditive, prônée pour l'enseignement des langues (entre autres domaine d'intervention) par Petar Guberina. Ainsi, Hervé Platel (2007) nous explique que loin d'être compartimentées, les « régions » du cerveau communiquent entre elles, de telle sorte que les phénomènes musicaux et langagiers, même si les rapports qu'ils entretiennent sont très difficiles à suivre et à repérer, ne doivent pas être pris dans un rapport d'opposition musique/langage (Platel, 2017). De plus, il est parfaitement démontré, neuro-imagerie à l'appui, que sons de parole et sons de musique partagent des «régions communes » du cerveau : 
«La neuro-imagerie cognitive montre clairement que l'analyse par le cerveau d'informations liées à des sons de paroles ou de musique implique des régions communes du cerveau, en particulier des régions préfrontales comme l'aire de Broca. » (Platel, 2017 : p. 148 et schéma p. 145)

Il nous apporte également une précision importante pour le professeur de musique de langue dans la manière d'aborder l'apprentissage de la lecture : « la perception musicale engage les régions temporales de façon plus antérieure, alors que la perception du langage active des régions postérieures du lobe temporal. Il y a en revanche plus de recouvrement des régions pariéto-occipitales activées pour la lecture de partition et la lecture de mots » (Platel, 2010). Cerveaux musicien et linguistique se rejoindraient par conséquent davantage dans l'activité de lecture et il serait par conséquent intéressant d'accorder davantage à la lecture de mots la qualité de lecture musicale.

Enfin, Platel $(2010,292)$ nous invite, avec (Liégeois-Chauvel et al.,1998 ${ }^{6}$ ), à «aborder la perception de la musique dans toute sa complexité, en considérant ses multiples processus, chacun de ces processus pouvant être latéralisé différemment dans les hémisphères cérébraux ».

\subsection{Chaque « cerveau musicien » est unique : deux caractéristiques méconnues}

$\mathrm{Si}$ les non spécialistes en neurosciences que nous sommes ont tendance, malheureusement, à ne retenir que des généralités superficielles en matière de fonctionnement neurocognitif, il convient cependant de ne pas oublier simplement que chaque cerveau est unique. Dans le domaine de la perception musicale des langues, toutes les expériences vécues par l'apprenant dans sa vie scolaire, familiale, socioculturelle et professionnelle ont une influence sur la nature de ses réactions. Nous savons que certains apprenants sont naturellement plus visuels qu'auditifs ou vice versa (Aubin, 1996). Nous avons repéré pour cet article deux éléments que le professeur de musique de langue doit avoir à l'esprit.

\section{a. Émotion musicale : de 0 à l'infini...}

Il existe une pente abrupte pour un enseignant bien décidé à exploiter la perception de la musicalité de la langue qu'il enseigne, à conditionner la conscience musicale d'une langue non seulement de façon objective mais surtout activer la fibre subjective. Il existe des personnes qui ne ressentent aucune émotion face à la musique :

«Cette approche connexionniste, qui est aujourd'hui dominante dans les travaux de neuro-imagerie cognitive, nous permettra de rendre compte avec davantage de pertinence de la variabilité individuelle du fonctionnement cérébral, et de mieux comprendre pourquoi il existe par exemple des personnes chez qui la musique ne produit aucune émotion alors même qu'elles ressentent des émotions à partir d'autres sources. » (Platel, 2017 : 148).

\footnotetext{
${ }^{6}$ LIÉGEOIS-CHAUVEL, C., PERETZ, I., BABAI, M., LAGUiTTON, V. \& CHAUVEL, P. (1998). Contribution of different cortical areas in the temporal lobes to music processing. Brain, 121, 1853-1867.
}

XLinguae, Volume 14 Issue 4, October 2021, ISSN 1337-8384, eISSN 2453-711X 
Cette réalité, que nos divers passages par l'acoustique musicale ne nous avaient pas révélée, nous conduit à penser que cette absence d'émotion sera encore plus marquée ou en tout cas plus imprévisible face à l'audition de la musique d'une langue, de la musique de la langue française, même si cela reste à vérifier. La gestion des variations possibles sur l'échelle de l'émotivité musicale langagière entre les apprenants qui pourraient ne rien ressentir et ceux qui sont facilement (em)portés par la beauté, selon eux, de la musicalité du français produite qui plus est, par telle ou telle voix ou timbre de voix qui les enchante, par la simple mélodie d'une phrase ou d'une expression, est un défi difficile à relever.

\section{b. Différence musicale entre une écoute masculine et une écoute féminine}

Nous savons combien les manières d'écouter et la durée de la qualité de l'écoute ne sont jamais exactement les mêmes d'un apprenant à l'autre. Or il y aurait des caractéristiques communes aux hommes et des caractéristiques communes aux femmes quiplaceraient naturellement hommes et femmes dans des modalités d'écoute différentes. En effet, selon les recherches neuropédagogiques présentées par Jacques Belleau, les femmes et les hommes n'écoutent pas de la même manière :

«On observe (...) que les hommes et les femmes écoutent différemment. Les hommes se servent du lobe temporal de l'hémisphère gauche associé aux fonctions d'écoute et de parole alors que les femmes ajoutent le lobe temporal de l'hémisphère droit utilisé pour jouer de la musique et se situer dans l'espace. Cette observation est importante, car elle nous dit que les femmes s'attardent aussi à un autre aspect de la parole, celui associé au rythme. Le rythme fait partie du message, il génère des émotions, donnent des indications sur les intentions. » (Belleau, $2015: 16$ ).

Cette différence observée, susceptible à n'en pas douter de créer des débats nourris et spontanés, est particulièrement intéressante pour notre spécialité musicale. Elle laisse à penser que les femmes seraient naturellement plus tournées que les hommes vers l'écoute musicale d'une langue puisque sa perception de la parole rejoint celle de la pratique d'un instrument de musique. Elle serait de plus prédisposées à l'étude du rythme d'une langue dont elle aurait une perception plus accentuée.

\subsection{Déductions pour le professeur de musique de langue-culture française}

Nous pouvons déduire de ce bref passage par les neurosciences cognitives musicales d'expression scientifique francophone quelques points capables d'alimenter et de canaliser des démarches méthodologiques et pédagogiques.

a. Il existe une proximité perceptive des sons de langue parlée et des sons de musique instrumentale, les sons de langue pouvant être musicalement perçus ;

b. Le fait de soumettre à l'analyse du cerveau d'un apprenant des sons de langue parlée, conformément à leur qualité et identité de sons musicaux, non seulement ne semble pas pouvoir être «nocif» ou constituer «une faute contre le cerveau» pour son bon développement cognitif mais au contraire, permet probablement de mobiliser et structurer davantage son potentiel neuronal musical ; 
c. Les systèmes et programmes éducatifs qui, malgré une orientation théoriquement tournée vers la transversalité et sauf expérience exceptionnelle, tendent dans la réalité quotidienne, à cloisonner les enseignements langagiers, plaçant d'un côté, les matières qui ne seraient que linguistiques et non musicales, de l'autre, les matières qui ne seraient que musicales, freinant ce que les neurosciences appellent la «neuroplasticité fonctionnelle» du cerveau, celle des apprenants en musique du français dans notre cas.

d. Par conséquent, cette tendance à séparer assez radicalement langues et musiques, surtout lorsque l'enfant avance en âge et en scolarisation, sans considérer leurs points communs et leurs différences, est contraire à la fonctionnalité du cerveau et ne serait pas naturelle.

e. Un professeur de langue qui prendrait auprès de ses élèves l'identité de professeur de musique de langue serait plus proche du fonctionnement du cerveau de ses élèves plutôt que celui qui n'enseignerait constamment qu'une langue.

f. Si l'apprenant en musique du français et en langue-culture française possède un cerveau musicien en cours de structuration, l'idéal serait de parvenir à le cultiver alors qu'il est le plus souvent ignoré et favoriser équilibre et plasticité entre les cerveaux musiciens et linguistes.

g. Si l'on peut parler de cerveau musicien pour un apprenant en musiques de langue, il serait important de considérer davantage si et comment l'intelligence musicale de chaque apprenant peut être favorisée.

Il serait alors intéressant de savoir si les techniques de neuro-imagerie cognitive ou autres techniques utilisées actuellement pourraient nous montrer comment le cerveau d'un apprenant en français réagit en faisant varier l'identité et les représentations mentales d'une langue produite sur le mode parlé :

a. À l'écoute des éléments sonores et musicaux de la langue si le sujet écoutant considère que l'objet entendu est une langue ;

b. À l'écoute des éléments sonores et musicaux de la langue si le sujet écoutant considère que l'objet entendu est une matière sonore ;

c. À l'écoute des éléments sonores et musicaux de la langue si le sujet écoutant considère que l'objet entendu est une musique de langue à part entière ;

d. À ces trois écoutes $(\mathrm{a}, \mathrm{b}, \mathrm{c})$ mais avec une langue que l'on comprend très bien, moyennement, un peu, pas du tout ;

e. À ces mêmes écoutes mais avec une langue très éloignée culturellement du sujet écoutant ;

f. Etc.

De même, il faudrait soumettre à ces écoutes divers discours produits dans différents contextes et situations de communication présentant des schémas musicaux différents, avec des voix enregistrées mais aussi avec des voix in vivo. 
L'apprenant en langue possède déjà, en tant que sujet parlant, un cerveau musicien. Il a une forte probabilité de le développer, surtout s'il est ou devient un apprenant en musique de langue, au plus près de la réalité. Cette identité lui offrant une base et un cadre mental pour orienter son écoute musicale et développer les régions du cerveau qui s'y prêtent.

\section{Conclusion}

Les deux questions qui nous ont servi de fil conducteur et ont porté un regard, en 2021, sur l'acoustique musicale et les neurosciences musicales ont abouti au recueil de plusieurs données pertinentes pour la Didactologie-didactique de la musique de la langue-culture française.

La mise en relief des qualités musicales des sons de la langue permet de présenter l'objet d'enseignement-apprentissage, indépendamment des goûts personnels et préférences en matière d'esthétique langagière, conformément à la réalité physique : une langue est constituée de sons musicaux combinés rythmiquement et mélodiquement de manière unique, ce qui lui confère l'identité de musique, de système musical faisant partie d'un genre musical différent des autres langages musicaux existants. La nature musicale des sons et combinaisons sonores de langue et de l'instrument à vent que chaque apprenant en langue donc en musique de langue doit apprendre à jouer différemment est largement posée et démontrée, de telle sorte que l'enseignant est avant tout un enseignant en musique et possède une triple identité à gérer : enseignant en musique, en langue, en culture. Il s'agit alors de parvenir à concilier ces identités musicale, langagière, linguistique et culturelle qui se retrouvent en cours de français en veillant à ce qu'aucune ne soit mise en veilleuse ou sousentendue.

Pour la Didactologie-didactique de la musique de la langue-culture française, la consultation périodique de l'acoustique musicale et des neurosciences ne permet pas de déceler une "contre-indication » dans le conditionnement didactique volontaire d'une perception musicale de la langue cible / musique cible. Cette pratique est d'autant plus indiquée que la connaissance du cerveau évolue vers la plasticité, la modularité avec des régions communes langage-musique et une grande capacité d'activation neuronale pour la formation d'un « cerveau musicien ». En l'absence de voix dissonante sur la nature musicale d'une langue, nous considérons que parmi les trois identités (musique-langue-culture), la branche enseignement-apprentissage de la musique d'une « langue nouvelle » est celle qui doit être prioritaire dans la mesure où non seulement elle garantit à une langue d'être une «langue vivante » mais le rôle déterminant d'aider l'apprenant à parler en français à son tour de la manière la plus juste, agréable, esthétique possible lui revient. Le fait de se centrer sur des modalités d'écoute variées et d'évoluer dans le cadre de Didactologie-didactique de la musique de la langue-culture française compte parmi les facteurs de progression de la qualité des performances expressives et communicatives des apprenants.

Cette étude vient confirmer ce que nous avons souvent observé : la conscience musicale de la langue parlée est plus élevée dans les disciplines musicales que les disciplines linguistiques, ces dernières ayant une tendance à s'en éloigner, hormis lorsque la langue devient poétique ou est mise en musique.

Il est certain que l'ancrage maximal de concepts et d'éléments musicaux de langue et de la langue-culture française doit être poursuivi. Il présente le grand avantage de 
pouvoir travailler à la fois et de façon continue sur le développement de la perception musicale de l'apprenant et sur l'enrichissement de sa culture musicale.

\section{Bibliographic references}

AUBIN, S. 1996. La didactique de la musique du français : sa légitimité, son interdisciplinarité. Thèse de doctorat dirigée par Jacques Cortès, Université de Rouen, France ; 1997. Villeneuve d'Ascq : Presses Universitaires du Septentrion. ISBN 9782729517205 .

AUBIN, S. 2008. Pourquoi enseigner les musiques de langue-culture ? In : Synergies Espagne, n. 1, pp. 41-46. ISSN 2260-6513. Available online: https://gerflint.fr/Base/Espagne1/aubin.pdf

BELLEAU, J. 2015. Neuropédagogie : cerveau, intelligences et apprentissage. Centre de documentation collégiale, Éducation et enseignement supérieure, Québec. Available online: http://eduq.info/xmlui/handle/11515/19677

BILLIERES, M. 2021. Au son du fle. Available online: https://www.verbotonalephonetique.com/

CORRAL FULlA, A. - AUBIN, S. 2017. De ce dixième numéro. In : Musiques, théâtre et didactique de la langue-culture française, Synergies Espagne, n. 10, pp. 1116. ISSN 2260-6513. Available online:

https://gerflint.fr/Base/Espagne10/corral_aubin.pdf

CORTÈS, J. 2018. Langue-culture française et neurosciences cognitives. Essai de bilan en 2018. In : Essais francophones, vol. 5. Sylvains-les-Moulins : GERFLINT. ISSN 2268-1582. Available online:

https://gerflint.fr/Base/Essais_francophones/essais_francophones_vol_5_2018.pdf

DEBERGH, C. 2017. L'anacrouse comme modèle pour appréhender le travail phonétique en langue française. In : Synergies Espagne, n. 10, pp. 19-37. ISSN 2260-6513. Available online: https://gerflint.fr/Base/Espagne10/debergh.pdf

GALAZZI, E. 1997. Physiologie de la parole et enseignement de la prononciation fin $19^{\mathrm{e}} /$ début $20^{\mathrm{e}}$ siècles. In : Documents pour l'histoire du français langue étrangère ou seconde, n. 19, Juin 1997, SIHFLES, pp. 166-183. ISSN 0992-7654.

GALISHNIKOVA, E. M. - BAKLASHOVA, T. A. - SAKHABIEVA, K. R. 2018. Formation od professional identity: context of training graduates in pedagogy. In: 4th International forum on teacher education (IFTE 2018), vol. 45, pp. 804-815.

GUILLEMIN, A. 1882 ( $3^{\mathrm{e}}$ éd.). Petite encyclopédie populaire des sciences et de leurs applications. Le son. Notions d'acoustique physique et musicale. Paris : Librairie Hachette. Available online:

https://gallica.bnf.fr/ark:/12148/bpt6k201314f.pdf Gallica, BNF

LANDERCY, A. - RENARD, R. 1982, 1977. Éléments de phonétique. Mons : CIPA. ISBN 13: 9782870885093

LECHEVALIER, B. - PLATEL, H. - EUSTACHE, F. 2010. Le cerveau musicien. Neuropsychologie et psychologie cognitive de la perception musicale. De Boeck Supérieur, «Questions de personne ». ISBN 9782804162801.

DOI : $10.3917 /$ dbu.leche.2006.01. Available online:

https://www.cairn.info/le-cerveau-musicien--9782804162801.htm

LEIPP, E. 1989, 1971. Acoustique et musique. Paris : Masson. ISBN 978-2-91125639-4.

LHOTE, E. (Dir.) 1987. À la découverte des paysages sonores des langues. Annales littéraires de 1'Université de Besançon, 353. Paris: Les belles lettres. ISBN 9782251603537.

XLinguae, Volume 14 Issue 4, October 2021, ISSN 1337-8384, eISSN 2453-711X 
PIERCE, J.R. 1983. Le son musical : musique, acoustique et informatique. Traduit de l'anglais par F. Berquier. Paris: Pour la science, coll. L'univers des sciences. ISBN 2-9029-1835-6.

PLATEL, H. 2010. Anatomie fonctionnelle de la perception et de la mémoire musicale. In : Bernard Lechevalier et al., Le cerveau musicien, pp. 291-303. ISBN 9782804162801. DOI : 10.3917/dbu.leche.2006.01.0291. Available online: https://www.cairn.info/le-cerveau-musicien--9782804162801-page-291.htm

PLATEL H. 2017. L'étude du cerveau nous aide-t-elle à mieux comprendre l'impact de l'art sur nos vies ? In : Nectart, vol. 1, n.4, pp. 144-151. ISSN 2429-2877. DOI : 10.3917/nect.004.0144. Available online: https://www.cairn.info/revue-nectart-20171-page-144.htm

RENARD, R. 1979, 1971. La méthode verbo-tonale de correction phonétique. Mons : Didier Erudition/CIPA. ISBN 2-86460-089-7.

RIVENC, P. 2013. Charles Bally et Petar Guberina, inspirateurs audacieux de la didactique moderne des langues. In : Synergies Espagne, n. 13. pp. 145-159. ISSN 2260-6513. Available online:

https://gerflint.fr/Base/Espagne6/Article10Paul_Rivenc.pdf

SIGIRCI, İ, 2018. Méthodes pour la correction des productions orales chez des apprenants turcs. In : Synergies Turquie, n. 11, pp. 53-66. ISSN 2257-8404 Available online: https://gerflint.fr/Base/Turquie11/sigirci.pdf

TROCME-FABRE, H. 1987. J'apprends, donc je suis: Introduction à la neuropédagogie. Paris : Les éditions d'organisation. ISBN 978-2708108608.

VALEEVA, R. - BAKLASHOVA, T. - LATYPOVA, L. 2018. Management of novice teachers' induction to the profession: Modernization of the russian school methodological system In: Journal of E-Learning and Knowledge Society, vol.14, n.2, pp. 39-50

ZAREKAR, F. - RAHMATIAN, R. 2017. Le degré d'influence de la verbo-tonalité lors des activités d'écoute et de lecture auprès des apprenants iraniens du français langue étrangère. In: Synergies Europe, n. 12, pp. 89-104. ISSN 2260-653X. Available online: https://gerflint.fr/Base/Europe12/zarekar_rahmatian.pdf

Words: 6727

Characters: 44611 (24,80 standard pages)

Prof. Dr Sophie Aubin

Department of French and Italian

Faculty of philology, Translation and Communication

Universitat de València

46010 València

Spain

sophie.aubin@uv.es

ORCID : 0000-0001-7425-3324 